Génét. Sél. Evol., 1985, 17 (3), 331-340.

\title{
Effect of alcohol and competition levels on viability of eye colour mutants of Drosophila melanogaster
}

\author{
Carmen NÁJERA and J.L. MÉNSUA \\ Departamento de Genética. Faculdad de Ciencias Biologicas \\ Universidad de Valencia, 50 Dr. Moliner, Burjassot, Valencia, Spain
}

\begin{abstract}
Summary
In order to test the influence of ethanol on viability of eye mutant strains from a cellar population, a factorial analysis was carried out for each mutant, using 4 alcohol concentrations, 2 levels of competition and 3 genotypes. The competition level-alcohol concentration interaction gives rise to better viability in the mutant strains, especially in the heterozygotes. It seems that eye colour mutants have a better viability in the special conditions of the cellar, which could explain their high frequency in this habitat.
\end{abstract}

Key words : D. melanogaster, viability, ethanol, competition level, eye colour mutant.

\section{Résumé}

Effet de l'alcool et de la surpopulation sur la viabilité de mutants de la couleur des yeux de Drosophila melanogaster

Pour étudier l'influence de l'éthanol sur la viabilité de souches mutantes pour la couleur des yeux et extraites d'une cave à vin, une analyse factorielle a été réalisée pour chaque mutant, avec 4 concentrations d'alcool, 2 niveaux de compétition larvaire et 3 génotypes. L'interaction alcoool-surpopulation conduit à une meilleure viabilité des souches mutantes, spécialement pour les hétérozygotes. Il semble que les mutants ont une meilleure viabilité dans l'environnement particulier des caves, ce qui peut expliquer leur fréquence élevée dans cet habitat. yeux.

Mots clés : D. melanogaster, viabilité, éthanol, surpopulation, mutants de couleur des

\section{Introduction}

The selectionist versus neutralist controversy on the maintenance of gene variation in natural populations has been debated for many years (see reviews of LEwONTIN, 1974 ; Ner, 1975). 
From a selective point of view there are several proposed explanations, which are not mutually exclusive. DobzhansKy $(1952,1970)$ proposed that the principal cause of maintenance is heterosis while others (Ayala \& Cambell, 1974 ; KoJima, 1971 ; Petit, 1968) consider frequency-dependent selection to be important. Another mechanism which could lead to a stable polymorphism is the diversification of ecological niches (LEVENE, 1953).

Although D. lebanonensis has an even higher tolerance to ethanol (DAviD et al., 1979), D. melanogaster is also remarkable because of its high alcohol tolerance especially in populations from the temperate zone (McKenzIE \& Parsons, 1972; David \& BocQUET, 1974, 1977). This peculiarity is of ecological importance because the species is capable of developing in ethanol-rich habitats such as wine cellars.

Comparisons of natural populations of Drosophila melanogaster from wineries and non-winery sites suggest that the former populations have a higher ethanol tolerance than the latter (McKenzie \& Parsons, 1974; McKenzie \& McKechnie, 1978; Hickey \& McLean, 1980).

In laboratory experiments, in situations of choice, larvae of $D$. melanogaster were shown to migrate to agar containing high concentrations of ethanol instead of pure agar, in contrast with D. simulans larvae (PARSONS \& KING, 1977).

In a previous analysis of eye colour mutants carried out in cellar and vineyard populations (NAJERA, unpublished data), the number of eye colour mutants per female was $0.628 \pm 0.040$ (93 mutants of 148 wild females analysed) in a cellar (Requena, Valencia, Spain) and $0.331 \pm 0.043$ (40 mutants of 121 females analysed) in a vineyard $4 \mathrm{~km}$ away from the cellar.

In order to examine the influence of alcohol on the viability of eye coulour mutants obtained in a wine-cellar and to bring some light on the maintenance of variability in this population, an experiment with 3 factors (strains, alcohol concentrations, competition levels) was designed.

\section{Material and methods}

Wild-caught females of $D$. melanogaster captured in a cellar in Requena, Valencia (Spain) were used to establish isofemale strains. Four of them were eye colour mutants : an allele of sepia $\left(\mathrm{se}^{770}\right)$; an allele of safranin $\left(\mathrm{sf}^{77 \mathrm{~m}}\right)$; an allele of cardinal $\left(\mathrm{cd}^{770}\right)$ and a multichromosomal strain ( $\left.\mathrm{cd}^{7 \tau o}, \mathrm{cn}^{770}, ?\right)$. Another one (wild strain, + ) did not segregate in $F_{2}$ any mutant at all (analysing 11 pair-matings in the $F_{1}$ flies).

The strains have been maintained in mass culture for 4 years.

Three factors were tested :

a) Two levels of competition for food $(25 \mathrm{cc}$ or $2 \mathrm{cc}$ of agar-sugar-corn meal medium).

b) Four levels of ethanol concentration ( 0 p. 100, 5 p. 100, 10 p. 100 and 20 p. 100). The alcohol was added to standard medium.

c) Three different genotypes (mutant homozygote, wild homozygote and heterozygote for each strain - a total of 9 different genotypes). 
All the vials were supplemented with live yeast.

The flies were left for a maximum of 12 hours in the usual medium (RoBERTSON, 1960) and afterwards the eggs were collected. One hundred eggs were placed in each of the 720 vials, since 10 replicates were made for each factor. The number of adults was counted.

All the experiments were carried out at $25 \pm 1^{\circ} \mathrm{C}$ temperature in a themoregulated chamber in which the relative humidity oscillated between 60-65 p. 100 with permanent light.

A three way factorial Anova (SoKal \& Rohlf, 1969 was made, using the arc sine transformation

$$
\operatorname{arc} \text { sine } \sqrt{\frac{(\mathrm{n}+3 / 8)}{(\mathrm{N}+3 / 4)}}
$$

where $n$ is the number of emerged adults and $N$ the number of seeded eggs. The same arc sine transformation was used for the sex ratio where $n$ is the number of males and $N$ the total.

\section{Results}

Table 1 shows the average viability of the 10 replicates for each of the strains studied in each medium and each situation.

The viability is higher for $25 \mathrm{cc}$ medium compared with $2 \mathrm{cc}$ medium, and better in some strains ( $\mathrm{cd},+)$ than in others (se, sf). The viability of 3 of the 4 heterozygotes is similar in these conditions. The viability of the $+/$ multichromosomal heterozygote is rather lower.

Graphs for the 4 strains are shown in figure $1(a, b, c, d)$. The abscissa is used for alcohol concentration and the ordinate for viability of each mutant strain, of the wild strain and of its heterozygotes, differentiating the 2 competition levels. It can be observed that the wild strain has better viability in $25 \mathrm{cc}$ medium and without alcohol ; the viability of this strain decreases when the alcohol concentration increases.

In the $c d$ strain (fig. 1 a) there is practically no difference between homozygotes and heterozygotes. Viability is not much affected when the alcohol concentration is increased.

In the se strain (fig. $1 \mathrm{~b}$ ) viability of heterozygotes is always higher than that of homozygotes. In the experiment with 2 cc medium when the alcohol concentration is increased, viability is maintained in both homozygotes and heterozygotes.

In the $s f$ strain (fig. $1 \mathrm{c}$ ) a response very similar to the above strain is observed, although perhaps the correlation between competition levels and alcohol concentration is more evident, and an increase of viability in homo- and in heterozygotes is detected when the alcohol concentration is increased in $2 \mathrm{cc}$ medium. 


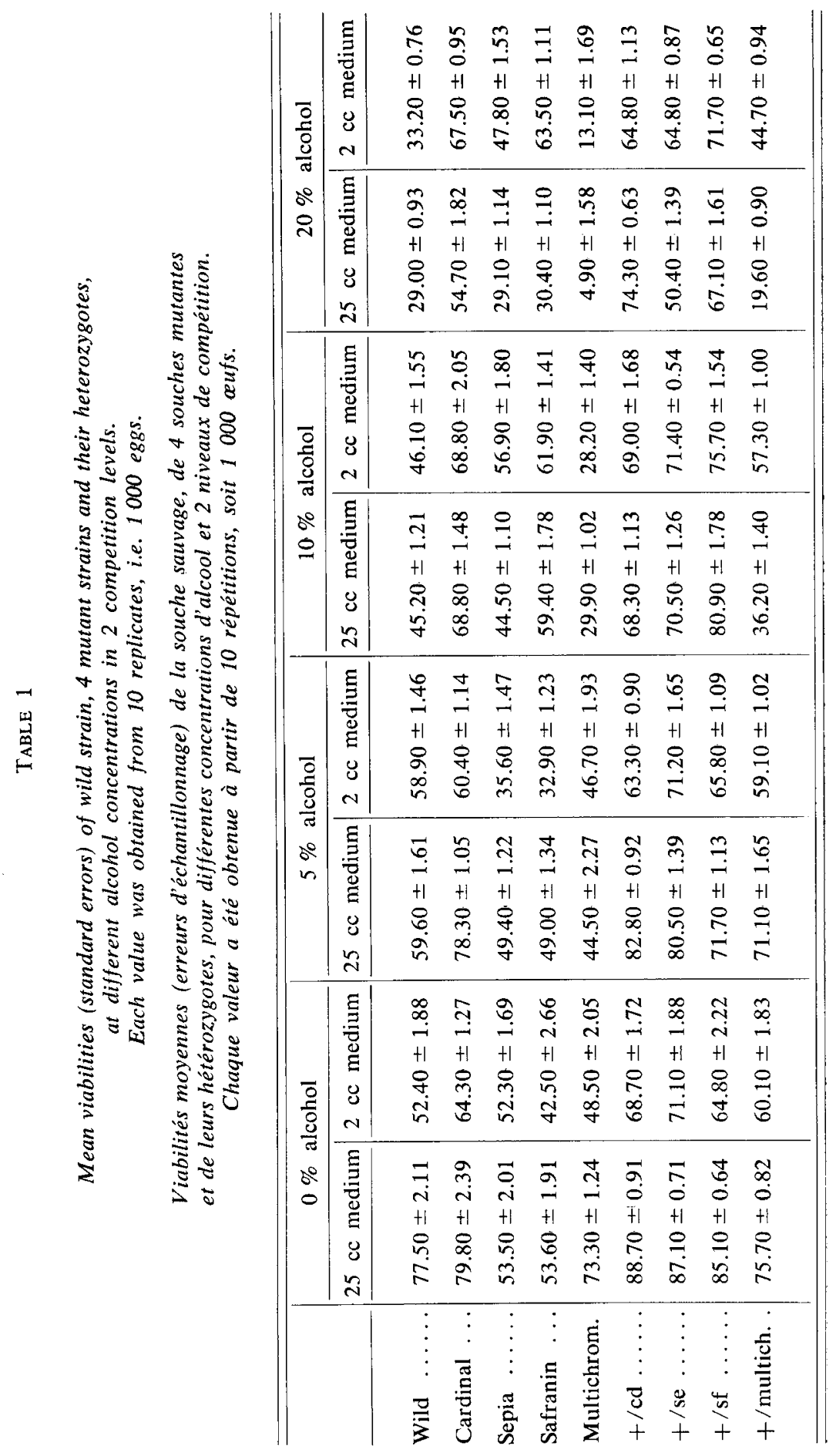



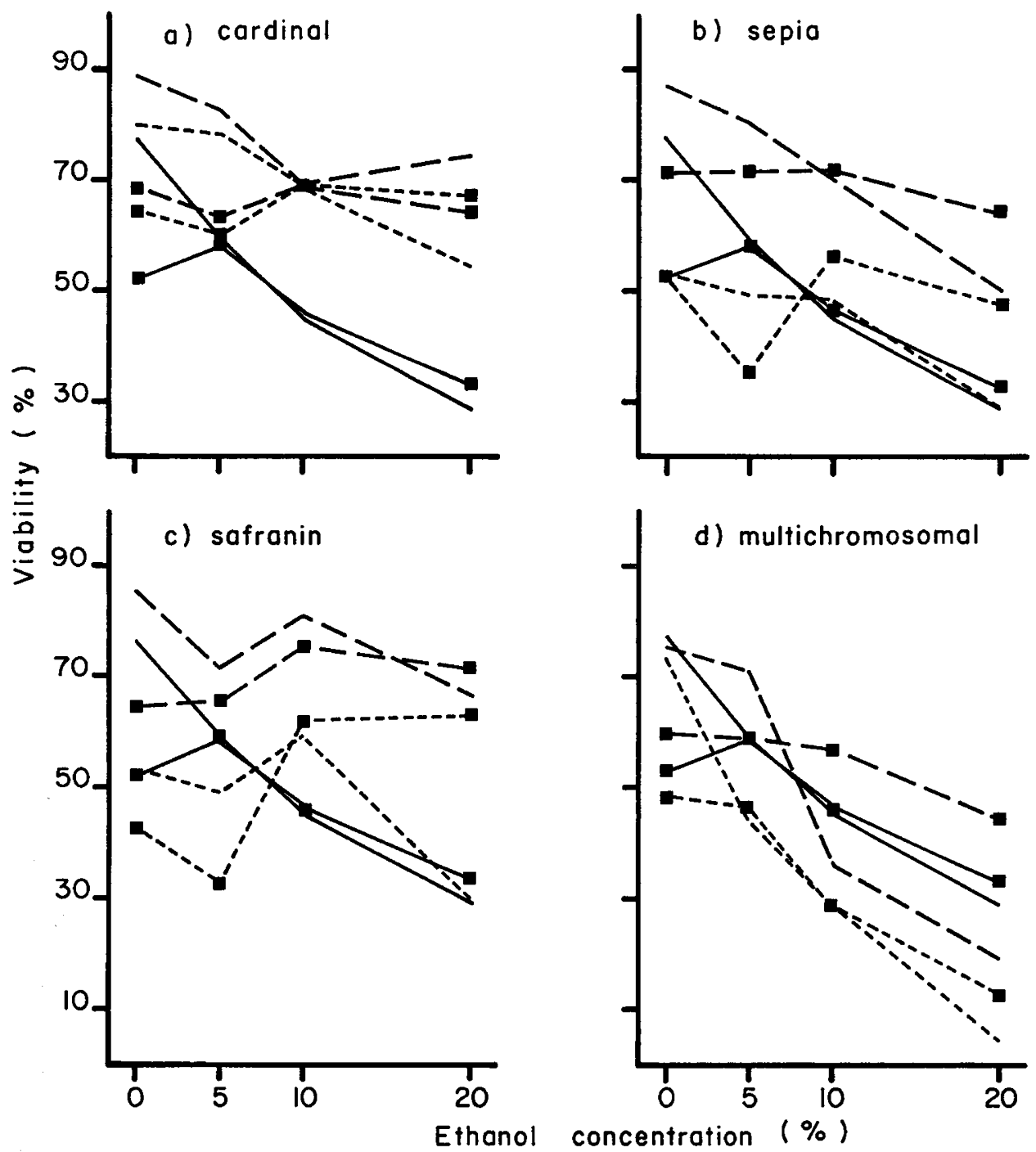

FIG. 1

Viability of the wild and mutant strains at different alcohol levels.

Viabilité des souches mutantes et de la souche sauvage aux différentes concentrations d'alcool dans le milieu.

Wild homozygotes

Homozygotes sauvages

.... Heterozygotes

Hétérozygotes

.... Mutant homozygotes

Homozygotes mutants

$25 \mathrm{cc}$ medium

Sans compétition

- 2 cc medium

Avec compétition 


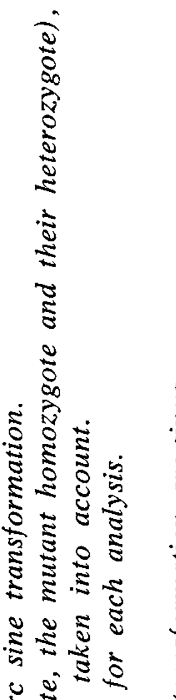

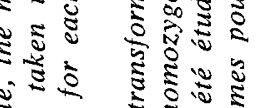

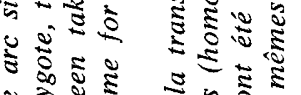

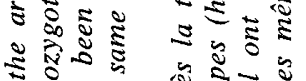
ง है केष भाग

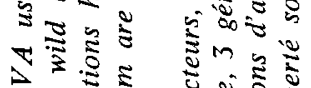

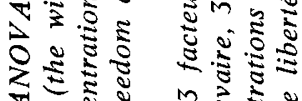

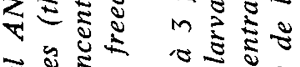
:

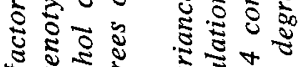

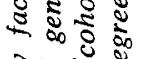
दि $\frac{0}{0} \frac{0}{0}$ ร.

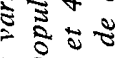

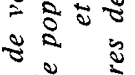

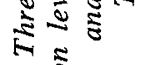

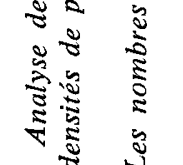

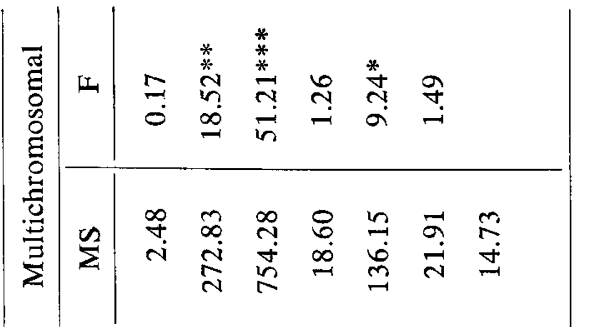

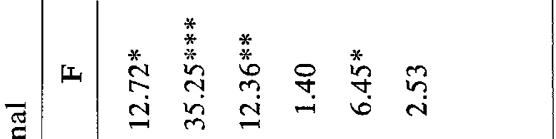

$$
\begin{aligned}
& \text { 胥 }
\end{aligned}
$$

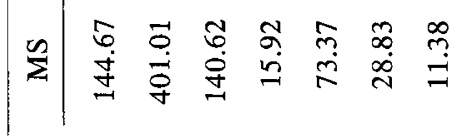

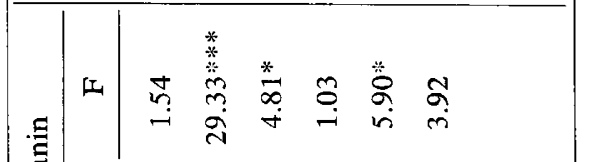

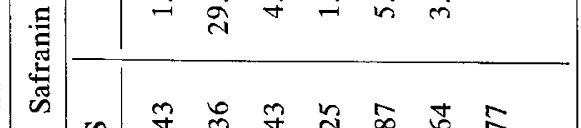

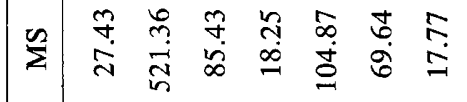

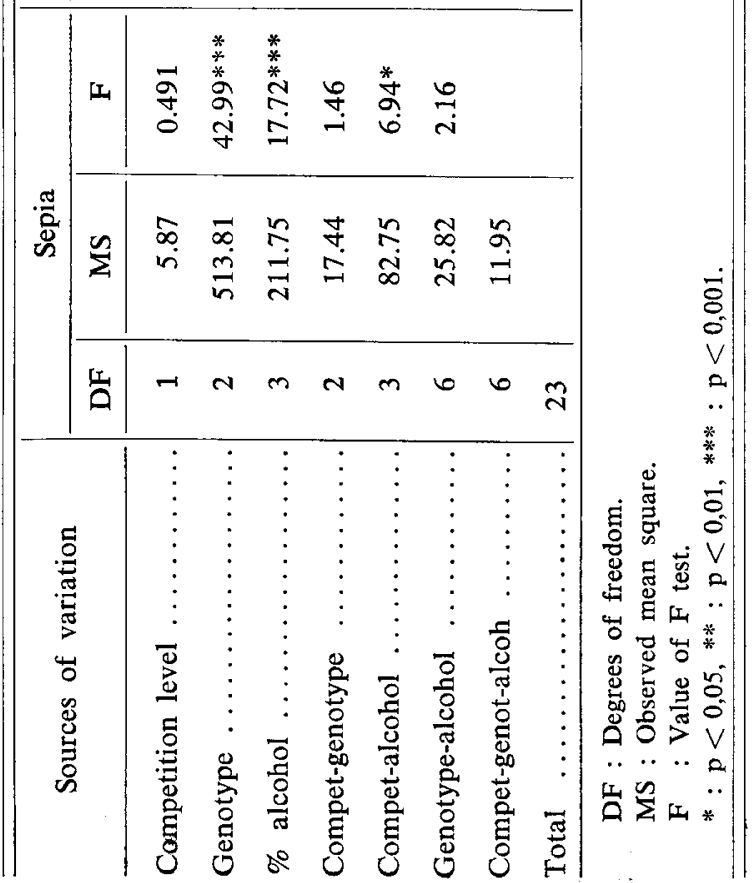


In the multichromosomal strain (fig. $1 \mathrm{~d}$ ) viability is very low and decreases greatly as the alcohol concentration is increased. It is always lower than in the wild strain except in heterozygotes with $2 \mathrm{cc}$ medium and high alcohol concentration, where a maintenance of viability can be observed as in the strains referred to above.

In order to check whether or not viabilities under different conditions are biased in favour of one or other sex, a factorial ANOVA (strains $X$ amount of food $X$ alcohol concentration) of sex ration $\left(\mathrm{n}^{\circ}\right.$ males/total) was carried out.

For the sex ratio, only the genotype factor $(F=4.26, \mathrm{P}<0.01)$ and the interaction between genotype and alcohol concentration $(\mathrm{F}=2.07, \mathrm{P}<0.05)$ are significant. There are no significant differences with regard to alcohol concentration, competition level or the interactions competition-genotype and competition-alcohol concentration.

This difference in the sex ratios among strains is explicable since in the multichromosomal strain, when the alcohol concentration is high $(20$ p. 100) the few individuals that emerge are females which leads to an appreciable imbalance in proportions.

The results of the 3 way factorial ANOVA are presented in table 2. The effects of genotype and alcohol are always significant but the competition level is significant only in the $c d$ strain. For the double interactions, only alcohol concentration-competition level interaction was significant in every strain.

In order to interpret the interaction between alcohol and competition level, the percentages of viability for the different genotypes at the 2 levels of competition were compared by means of a $t$ test. At 0 and at 5 p. 100 alcohol concentration there are always significant differences in favour of the low competition situation; at 20 p. 100 alcohol concentration all. genotypes differ significantly, always in favour of the high competition situation, whereas at 10 p. 100 alcohol concentration there are genotypes which differ significantly in favour of high competition situation (se, $+/$ multichromosomal) while one differs significantly in favour of a low competition situation $(+/ \mathrm{sf})$, and the remainder do not differ significantly.

The same $t$ test was carried out to compare the percentages of viability between the homo- and heterozygote genotypes in the different conditions. All the heterozygotes gave viability means higher than both homozygotes, with two exceptions: $+/$ cd versus cd and $+/$ multichromosomal versus wild.

\section{Discussion and conclusion}

The responses of larvae and adults to ethanol have been investigated by many authors. ARQUES \& DUARTE (1980) found an increase of larval viability at low ethanol concentrations. Parsons \& Spence (1981) found that at low concentrations ethanol is utilized as a resource without detrimental effect while at higher concentrations, above a certain threshold concentration, noxious effects increase and ultimately prevail. Primary alcohols can function as food components instead of toxic compounds on many occasions.

The adaptation process of Drosophila melanogaster to ethanol containing resources may mainly correspond either to a necessity for detoxification or to a need for nutrition. 
ADH is the enzyme in charge of metabolizing ethanol, but ADH activity and ethanol utilization as a resource are not obligatorily interdependent (VAN HERREWEGE \& DAVID, 1980, 1984).

Ethanol is a strong selective factor in favour of the $\mathrm{F}$ allele in laboratory populations (Gibson, 1970 ; Bulsma \& Van Delden, 1974 ; Morgan, 1975 ; OAKeshott, 1976 ; Barnes \& Birley, 1978 ; Cavener \& Clegg, 1981 ; Van Herrewege \& David, 1984) althought some controversial results have been reported (OAKESHOTT \& GIBSON, 1981 ; Ziolo \& PARSONS, 1982 ; OAKeshotT et al., 1983).

As regards natural populations, a higher $F$ frequency in winery populations comparing to others is reported by some authors (BRIscoe et al., 1975; HICKEY \& McLean, 1980) but denied by others (McKenzie \& Parsons, 1974 ; Mark et al., 1980).

The genotypes for the ADH locus of the strains used here, were all homozygous for the $\mathrm{F}$ allele, except the multichromosomal strain, which was homozygous for the $S$ allele. This strain has the worst viability at different alcohol concentrations.

Since the wild strain is homozygous for the $F$ allele as well as 3 of the mutant strains, and nevertheless, the viability of mutant strains is higher when alcohol concentration increases, it seems that the alcohol tolerance in these mutant strains is rather related with utilization as a nutritional resource than with a process of detoxification.

BiJlsma-Meeles (1979) found that alcohol concentrations decreased gradually by evaporation during the experimental period. If the alcohol concentration is high, its evaporation will give rise to toxic vapours which will be correlated to the amount of food. This could be a possible explanation for the interaction found between competition and alcohol concentration, which leads to improved viability in the eye colour mutants and still more in the heterozygotes. In low competition situations toxic vapours can affect viability of larvae as alcohol concentration increases. Nevertheless, in high competition situations there is practically no evaporation of alcohol, because it is consumed by the larvae before its evaporation.

Studies on the locus sepia (ANXOLABEHERE, 1976) have indicated that the main factor responsible for the maintenance of sepia in populations is frequency-dependent heterosis influenced by genetic background. In the present work the higher mean viability of the heterozygotes is clear in almost all the experimental conditions tested. Considering that mutant strains have the same origin as the wild strain, the higher viability of mutant strains when alcohol concentration increases, may be attributed to the mutant loci themselves. This could suggest a possible gene heterosis in eye colour mutants of cellar populations.

On the other hand, the higher viability of mutant strains when alcohol concentration increases in high level of competition, also leads to the consideration that the competitionalcohol concentration interaction might be a factor contributing to the maintenance of eye colour mutants variability in cellar populations.

Received April 17, 1984.

Accepted January 24, 1985. 


\section{References}

AnXolabehere D., 1976. Heterosis, overdominance and frequency-dependent selection in Drosophila melanogaster at the sepia locus. Evolution, 30, 523-534.

Arques L.V., Duarte R.G., 1980. Effect of ethanol and isopropanol on the activity of alcohol dehydrogenase, viability and life-span in Drosophila melanogaster and D. funebris. Experientia, 36, 828-830.

Ayala F.J., Cambell C.A., 1974. Frequency-dependent selection. Annual review of Ecology and systematics, 5, 115-137.

Barnes B.W., Birley A.J., 1978. Genetical variation for enzyme activity in a population of D. melanogaster. IV. Analysis of ADH activity in chromosome sustitution lines. Heredity, 40, 51-57.

BiJlsma-Meeles E., 1979. Viability in Drosophila melanogaster in relation to age and ADH activity of eggs transferred to ethanol food. Heredity, 42, 79-89.

Bijlsma-Meeles E., VAN Delden W., 1974. Intra and interpopulation selection concerning the ADH locus in D. melanogaster. Nature, 247, 369-371.

Briscoe D.A., Robertson A., Malpica J.M., 1975. Dominance at ADH locus in response of adult Drosophila melanogaster to environmental alcohol. Nature, 255, 148-149.

Cavener D.R., Clegg M.T., 1981. Multigenic response to ethanol in D. melanogaster. Evolution, 35, 1-10.

David J.R., Van Herrewege J., Monclus M., Prevosti A., 1979. High ethanol tolerance in two distantly related Drosophila species; A probable case of recent convergent adaptation. Comp. Biochem. Physiol., 63 C, 53-56.

David J., Bocquet C., 1974. L'adaptation génétique à l'éthanol : un paramètre important dans l'évolution des races géographiques de Drosophila melanogaster. C.R. Acad. Sci., Paris, 279, 1385-1388.

David J., Bocevet C., 1977. Genetic tolerance to ethanol in Drosophila melanogaster : increase by selection and analysis of correlated responses. Genetica, 47, 43-48.

Dobzhansky T., 1952. Nature and origin of heterosis. In : GoweN J. (ed.), Heterosis, 218-223. Iowa State College Press.

Dobzhansky T., 1970. Genetics of the Evolutionary process. 505 pp., Columbia University Press, New York.

Gibson J., 1970. Enzyme flexibility in Drosophila melanogaster. Nature, 227, 959-960.

Hickey D.A., McLean M.D., 1980. Selection for ethanol tolerance and alcohol dehydrogenase allozymes in natural populations of Drosophila melanogaster. Genet. Res., 36, 11-15.

KoJima K., 1971. Is there a constant fitness value for a given genotyppe? No! Evolution, 25, 281-285.

LEVENE H., 1953. Genetic equilibrium when more than one ecological niche is available. Am. Nat., 87, 331-333.

Lewontin R.C., 1974. The Genetic Basis of Evolutionary Change. 346 pp., Columbia Univ. Press, New York.

Marks R.W.. Brittnacher J.G., McDonald J.F., Prout T., Ayala F.J., 1980. Wineries, Drosophila, alcohol and ADH. Oecologia, 47, 141-144.

McKenzie J.A., Parsons P.A.. 1972. Alcohol tolerance : An ecological parameter in the relative success of Drosophila melanogaster and Drosophila simulans. Oecologia (Berl.), 10, 373-388.

McKenzie J.A., Parsons P.A., 1974. Microdifferentiation in a natural population of Drosophila melanogaster to alcohol in the environment. Genetics, 77, 385-394.

McKenzie J.A., McKechnie S.W., 1978. Ethanol tolerance and the ADH polymorphism in a natural population of Drosophila melanogaster. Nature, 272, 75-76.

Morgan P., 1975. Selection acting directly on an enzyme polymorphism. Heredity, 34, 124-127.

NeI M., 1975. Molecular Population Genetics and Evolution. 288 pp., North Holland, Amsterdam. 
OAKeshott J.G., 1976. Selection at the alcohol dehydrogenase locus in Drosophila melano. gaster imposed by environmental ethanol. Genet. Research, 26, 265-274.

OAKeshotT J.G., Gibson J.B., 1981. Is there selection by environmental ethanol on the alcohol dehydrogenase locus in Drosophila melanogaster? In : Genetic studies of Drosophila populations, 103-120. Aust. Nat. Univ. Press, Canberra.

OAKeshott J.G., Wilsons R., Gibson J.B., 1983. An attempt to measure selection coefficients affecting the alcohol dehydrogenase polymorphism in Drosophila melanogaster population maintained on ethanol media. Genetica, 61, 151-159.

Parson P.A., Spence G.E., 1981. Ethanol utilization : threshold differences among three Drosophila species. Am. Nat., 117, 568-571.

Petit C., Anxolabehere D., 1968. Frequency dependent selection and larval competition in Drosophila melanogaster. XII International Congress of Genetics, Tokyo, 19-28 Agosto, 1968. Abstracts of contributed papers $1: 228$. The Sciences Council of Japan. Ueno Park, Tokyo, Japon.

Robertson F.W., 1960. The ecological genetics of growth in Drosophila. I. Body size and development time on different diets. Genet. Res., 1, 288-340.

Sokal R.R., RohlF F.J., 1969. Biometry. 776 pp., Freeman W.H. and Company, San Francisco.

Van Herrewege J., David J.R., 1980. Alcohol tolerance and alcohol utilization in Drosophila : partial independence of two adaptive traits. Heredity, 44, 229-235.

Van Herrewege J., David J.R., 1984. Extension of life duration by dietary ethanol in Drosophila melanogaster : response to selection in two strains of different origins. Genetica, 63, 61-70.

Ziolo L.K., Parsons P.A., 1982. Ethanol tolerance, alcohol dehydrogenase activity and ADH allozymes in Drosophila melanogaster. Genetica, 57, 231-237. 